\title{
The fluid gels: A research review
}

\author{
Ke Zhang, ${ }^{1, *}$ \\ ${ }^{1}$ School of Food and Biological Engineering, Hubei University of Technology, Wuhan, 430068, China
}

\begin{abstract}
Fluid gels were formed during the sol-gel transition of biological polysaccharide, which were damaged by the shear field and fails to form a static gel network, and the resulting suspension of gel particles were also called shear gel. Modern food design usually requires both deliciousness and health. The special physical properties of fluid gels allow foods with reduced sugar and salt to maintain good texture and physical properties, so they are of great significance in modern product design. This article reviewed the preparation and latest research progress of fluid gels. It demonstrated their unique application prospects for the modern food industry.
\end{abstract}

\section{Introduction}

Fluid gels is a suspension of gel particles formed during the sol-gel transition process of polysaccharide, which is damaged by the shear field and fails to form a static gels network, which is also called shear gels[1-5]. Modern food design usually requires both deliciousness and health. The special physical properties of fluid gels enable reduced-sugar and salt-reduced foods to maintain favourable texture and physical properties, so it is of great significance in modern product design. In recent years, a large number of scholars have conducted a lot of research on fluid gels, and the similarities and differences between its microstructure and static gel have been characterized by rheometer, differential calorimetry scanner, scanning electron microscope, etc., revealing its unique texture properties and use value.

\section{Preparation of Fluid gels}

The materials used in the preparation of fluid gels are mainly agar, carrageenan, seaweed gum, and protein. The common feature of these polysaccharides is that they could form gels (cold gels or ion gels), and the preparation tools are mostly rheometer or jacket. Agitator and other Couette flow methods, rheometer can accurately control the shear rate and temperature rise and fall rate, which is of great significance for accurately studying the impact of shear rate and cooling rate on the structure and morphology of the fluid gels. Jacket stirring device has the characteristics of large output, both of which adopt the way of forming a shear field by the rotation of the rotor. The physical properties of the final particles can be adjusted by controlling the cooling rate.

\section{Fluid gels generation mechanism}

The generation mechanism of the fluid gels is that when the temperature is close to the transition point, the formation of the static network structure and the shearinduced rupture form a competitive relationship with each other, which will affect the final particle structure (size, shape). At high cooling rates, the gelation process dominates the applied shear rate and produces large irregularly shaped particles. When the conformational dynamics is more closely matched to the time scale of the applied shear rate (ie, at lower cooling rates), the input from the applied flow field increases, resulting in the formation of small spherical particles [1] [17]. Fluid gels are different from static network gels, resulting in fewer helical regions and smaller helix aggregates, as shown in Figure 1[1].

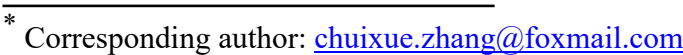



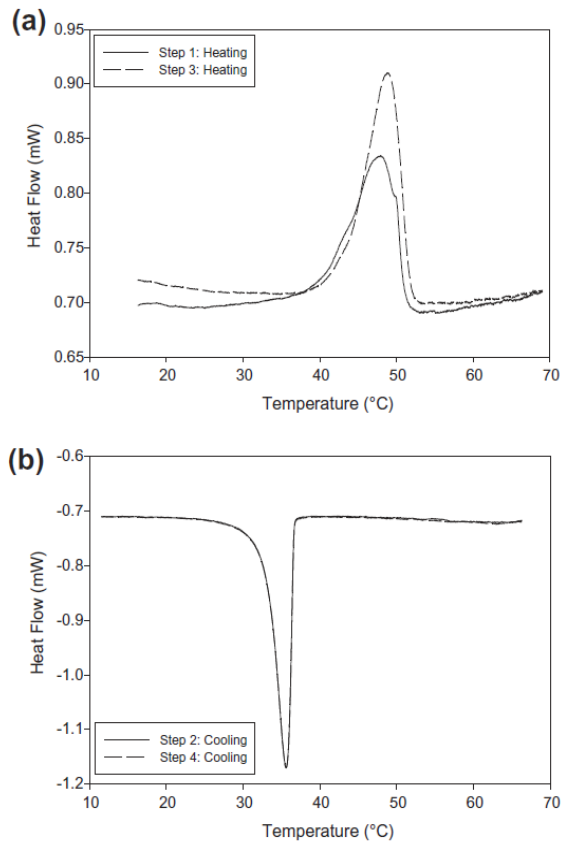

Fig. 1. DSC heating (a) and cooling (b) profiles for a product that was initially a fluid gel prepared from $0.5 \mathrm{wt} . \% \mathrm{jC}$ and 0.3 wt. $\% \mathrm{KCl}$. The first heating scan (Step 1) shows melting of a fluid gel; the second (Step 3), quiescent gel.

\section{Current research status on fluid gels}

The generation mechanism and morphological structure of the fluid gels have been extensively studied, which provides a rich theoretical basis for the design of food microstructures. The most work comes from the IT Norton research group. Norton IT [1] [8] discussed the role of biopolymer gels in the shear field, studied the similarities and differences between the aggregation caused by shearing in the gelation process and static gels, and proposed a structural model. Garrec et al. [1] discussed the formation and properties of a fluid gels produced by $\mathrm{k}$-carrageenan by applying shear during the gelation process. The thermal transitions of molten fluid gels and statically cooled gel are compared. Since shearing interferes with the molecular ordering process in the production process, the number of helices that determines the former is becoming less and less. They believe that for a smooth hydrodynamic gel sample, $>98 \%$ of the molecular sequence must be in a shear state. The applied shear force destroys the orderly arrangement of the molecules, forming fewer and fewer spirals, resulting in bridging between particles. Increased storage modulus and yield stress. The inter-particle bridges are irreversibly broken under shear. A. Gabriele et al. [2] [3] used a method to produce kappacarrageenan fluid gels using a certain geometry, flow pattern and cooling rate. A narrow size distribution is achieved by inducing spinodal decomposition under temperature quenching and controlled shear conditions, revealing the relationship between the increase in viscosity caused by particle formation and the concentration of $\mathrm{k}$-carrageenan. The lubrication behaviour of agarose fluid gel was studied, and the mechanism describing the process was proposed. This research has important implications for all processes involving soft contact between particles and surfaces, such as oral processing. Garrec DA et al. [4] used kcarrageenan lubricants formulated in the form of gel particles in solutions and suspensions (fluid gels) to test the tribology in the lubricating boundary state, thereby increasing the thickness The type of agent provides new insights into the structure of tribology. The polymer lubrication system shows that the elastic deformation of the friction surface is dominant, while the particle suspension is dominated by the particles that act as the mating surface and contact the rough surface. The results show that the tribology of the gelled particles largely depends on the elasticity of the particles, in which smaller deformed (irritating) particles will reduce surface contact, thereby reducing the coefficient of friction. The effect of particle volume fraction on the friction coefficient is related to the number of particles entrained in contact, and has nothing to do with particle-particle interaction or overall rheological behaviour. The effect of particle volume fraction on the friction coefficient is related to the number of particles entrained in contact, and has nothing to do with particle-particle interaction or overall rheological behaviour. Compaan AM et al. [5] used gellan gum fluid gels for 3D printing hydrogel structure. It is worth noting that the printing method used for mammalian cells can form cell-to-cell contact within a few hours after manufacturing. Cell viability was observed in the printed construct within a few days.

\section{Fluid gels application}

Fluid gels has been widely used in food manufacturing, biomedicine and other fields with its good biological affinity. Soft tribology has attracted more and more attention in the application of biotribology to approximate the skin/skin and oral contact environment. Mills $\mathrm{T}$ et al. [6] explored the tribological behavior of food and consumer products/model systems, and studied the relationship between the soft surface lubrication of agar-fluid gels and solvent quality. The pure solvent system shows a mode of reducing friction. When the solvent quality is changed, the lubricating behaviour of the fluid gels is different, and it is considered that it is mainly the effect of changing the gel structure. These effects can potentially be used to control food properties to enhance oral processing properties. Fluid gels have shown great potential in low-fat and low-calorie foods, as well as in the formulation of self-satisfaction foods. Bradbeer JF et al. [7] have controlled materials (the concentration of LA gellan gum and hydrochloric acid) and processes. The parameters of shear and cooling rate during the production of fluid gels can control the properties and size of individual particles and the interaction/bridging between them. Both the fluid gels and the acidified fluid gels produced by these production methods exhibit non-Newtonian shear thinning flow characteristics. They clearly show that the structure of LA gellan gum fluid gel can be controlled by its production process and exposure to acidic environments. 
When exposed to acidic conditions that mimic the environment of the stomach, the structure is still maintained in the same way as a solid static gel, which may increase the level of satiety. Sworn G et al. [9] prepared a gellan gum fluid gels system by cooling during a constant shear rate. Then, without disturbing the sample, an oscillatory shear measurement is used to determine structural properties. Using this method, the effects of ionic environment and gellan gum concentration on the transition temperature, viscosity and structural development of fluid gels systems were studied. Fluid gels have broad application prospects in food and medicine. The nasal mucosa provides a potentially good route for local and systemic drug delivery, however, the protective features of the nasal cavity make intranasal delivery challenging. The application of mucoadhesive polymers in nasal drug delivery systems enhances the retention time of drugs in the nasal cavity. Mahdi MH et al. [10] prepared a fluid gel by introducing shear force during the gelation process of gellan gum polymer, which can be expressed as a pourable viscoelastic fluid. The results show that by preparing a fluid gels of high acyl gellan gum, its unique rheological properties are sufficient to spray through a standard nasal sprayer. Fluid gels also have excellent interfacial properties. Lazidis A et al. [11] proved the ability of whey protein liquid gels to produce very stable foams and analyzed the mechanism of small and mobile proteins during the foaming process. The entity quickly diffuses to the interface and provides the necessary interfacial tension reduction to promote foam formation. Subsequently, larger protein particles fill the free space between bubbles and increase local volume viscosity, improving foam stability by preventing drainage. The whey protein fluid gels can produce the same amount of foam as untreated whey protein, but the stability is greatly improved. Farrés I F [12] studied the formation and properties of alginate liquid gels using in-situ calcium release and a defined shear field. The results showed that although the particle growth rate increased significantly during the formation process after reducing the shear rate, the interaction rate between particles after production was not affected by the shear amplitude. Longer polymer chains allow more possible calcium crosslinking sites on each chain, thereby increasing the formation of percolation networks and increasing the number of rheologically effective network crosslinks. And through the molecular weight and $\mathrm{CaCO} 3$ concentration to control the order kinetics and the resulting particle properties, it is possible to design and provide specific structures with required properties. Norton IT [13] reviewed the production, formation and properties of fluid gel for single and mixed biopolymer systems. The formation process of particles is a process of nucleation and growth, in which the viscosity of the evolved structure increases as the number and volume fraction of particles increase. The size (and number) of the particles depends on the shear rate and concentration. And established a theoretical model, which shows the important experimental parameters that need to be considered when understanding or even controlling the microstructure of a single or mixed biopolymer system.
Farrés I F et al. [14] have developed a new method for producing alginate fluid gels. By using the turbulent flow field generated in the pin agitator, fluid gels particles can be generated in a reproducible and controlled manner. For the alginate fluid gels produced, the average particle size produced by this method is less than $10 \mu \mathrm{m}$. It provides the possibility to construct microstructures with enhanced sensory properties. Due to the potential use of fluid gels in structured fluids, whey protein isolate (WPI) is used to prepare fluid gels. Moakes R J A et al. [15] prepared fluid gels by thermally induced gelation. The protein solution is prepared under controlled temperature and shear. It was found that the physical properties of the resulting gel particles (such as size and particle interaction) depend on the combination of shear force and heat history. Large discrete aggregates $(>120 \mu \mathrm{m})$ are obtained under low shear, and smaller aggregates $(<40 \mu \mathrm{m})$ are obtained under higher shear. This microstructure change in the particles leads to a change in the rheology of the suspension. All suspensions exhibited significant shear thinning behaviour, which was more pronounced for larger aggregates (originally made under low shear). Once in close contact (such as high volume fraction), the viscoelastic properties of the particle system are similar to pseudo-solid materials. In addition, the results show that at a given volume fraction, due to the degree of interaction between particles, the elasticity of the suspension varies depending on its original processing conditions. Patients with dysphagia usually prefer oral liquids for drug administration, but formulating sustained-release formulations can be challenging. Mahdi MH et al. [16] studied the potential way to achieve sustained release in oral liquids by using a fluid (shear) gel formed by introducing a shear field during the gelation process of the biopolymer formed by the gel. These fluid gels can act as pourable viscoelastic fluids, but retain the true gel micro/nano structure. It also proved that the fluid gel has the potential to be an oral liquid for children, which can prevent the release of ibuprofen in simulated gastric juice. Gabriele et al. [17] studied the formation, production and lubricating properties of liquid gels during the gelation of agarose and $\beta$-carrageenan. The results show that a fluid gels with a homogeneous particle size with an average particle size less than $5 \mu \mathrm{m}$ can be formed. The resulting fluid gels shows strong and highly recyclable particle bridges. Provides a model for the aggregation dynamics of these particles, providing information about the mechanisms involved. In recent decades, biopolysaccharides have been used in the food industry to provide structural stability and impart desired texture to a variety of products, including emulsions.

\section{Summary}

With the improvement of living standards and the intake of high-energy foods causing many health problems, foods with reduced sugar and salt are in strong demand. However, healthy foods usually have poor palatability. 
The design and modification of microstructures can innovate the types of foods and bring hope to the production of delicious and healthy foods. Fuid gels are a typical microstructure design. Scientists have done a lot of research and have developed methods that can control and manipulate the physical properties of structured components, and achieve changes in their microstructures by controlling process conditions. The raw materials can only rely on polysaccharides, and expanded to protein, greatly enriching the source of raw materials. Its applications are gradually diversified, from sugar-reduced and salt-reduced foods to elderly rehabilitation foods, from slow-release drugs to $3 \mathrm{D}$ printing, and more and more novel and more complex fluid gel structures are gradually produced. The design of microstructures requires knowledge in colloid science and physiology, sensory science and mechanical manufacturing. In the future, it should be more interdisciplinary and disciplinary cooperation to produce more updated theories and products, thereby making humans healthier and the delicious experience.

\section{References}

1. Garrec D A, Norton I T. Understanding fluid gel formation and properties[J]. Journal of Food Engineering, 2012, 112(3): 175-182.

2. Gabriele A, Spyropoulos F, Norton I T. Kinetic study of fluid gel formation and viscoelastic response with kappa-carrageenan[J]. Food Hydrocolloids, 2009, 23(8): 2054-2061.

3. Gabriele A, Spyropoulos F, Norton I T. A conceptual model for fluid gel lubrication[J]. Soft Matter, 2010, 6(17): 4205-4213.

4. Garrec D A, Norton I T. Kappa carrageenan fluid gel material properties. Part 2: Tribology[J]. Food Hydrocolloids, 2013, 33(1): 160-167.

5. Compaan A M, Song K, Huang Y. Gellan Fluid Gel as a Versatile Support Bath Material for Fluid Extrusion Bioprinting[J]. ACS applied materials \& interfaces, 2019, 11(6): 5714-5726.

6. Mills T, Koay A, Norton I T. Fluid gel lubrication as a function of solvent quality[J]. Food Hydrocolloids, 2013, 32(1): 172-177.

7. Bradbeer J F, Hancocks R, Spyropoulos F, et al. Low acyl gellan gum fluid gel formation and their subsequent response with acid to impact on satiety[J]. Food hydrocolloids, 2015, 43: 501-509.

8. Norton I T, Jarvis D A, Foster T J. A molecular model for the formation and properties of fluid gels[J]. International Journal of Biological Macromolecules, 1999, 26(4): 255-261.

9. Sworn G, Sanderson G R, Gibson W. Gellan gum fluid gels[J]. Food Hydrocolloids, 1995, 9(4): 265 271.

10. Mahdi M H, Conway B R, Smith A M. Development of mucoadhesive sprayable gellan gum fluid gels[J]. International Journal of Pharmaceutics, 2015, 488(1-2): 12-19.

11. Lazidis A, Hancocks R D, Spyropoulos F, et al. Whey protein fluid gels for the stabilisation of foams[J]. Food hydrocolloids, 2016, 53: 209-217.

12. Farrés I F, Norton I T. Formation kinetics and rheology of alginate fluid gels produced by in-situ calcium release[J]. Food Hydrocolloids, 2014, 40: 76-84.

13. Norton I T, Smith C G, Frith W J, et al. The production, properties and utilisation of fluid gels[M]/Hydrocolloids. Elsevier Science, 2000: 219-227.

14. Farrés I F, Douaire M, Norton I T. Rheology and tribological properties of $\mathrm{Ca}$-alginate fluid gels produced by diffusion-controlled method[J]. Food Hydrocolloids, 2013, 32(1): 115-122.

15. Moakes R J A, Sullo A, Norton I T. Preparation and characterisation of whey protein fluid gels: The effects of shear and thermal history $[\mathrm{J}]$. Food Hydrocolloids, 2015, 45: 227-235.

16. Mahdi M H, Conway B R, Smith A M. Evaluation of gellan gum fluid gels as modified release oral liquids[J]. International journal of pharmaceutics, 2014, 475(1-2): 335-343.

17. Gabriele A. Fluid Gels: formation, production and lubrication[D]. University of Birmingham, 2011. 